\title{
Immunohistochemical analysis of hippocampal butyrylcholinesterase: Implications for regional vulnerability in Alzheimer's disease
}

\author{
Katsuyoshi Mizukami, ${ }^{1,2}$ Hiroyasu Akatsu, ${ }^{3,4}$ Eric E. Abrahamson, ${ }^{5,7}$ Zhiping $\mathrm{Mi}^{5}$ and \\ Milos D. Ikonomovic $\mathbf{5 , 6 , 7}$
}

${ }^{1}$ Faculty of Health and Sport Sciences, ${ }^{2}$ Graduate School of Comprehensive Human Sciences, University of Tsukuba, Tsukuba, ${ }^{3}$ Department of Community-based Medicine, Nagoya City University Graduate School of Medicine, Nagoya, ${ }^{4}$ Choju Medical Institute, Fukushimura Hospital, Toyohashi, Japan, ${ }^{5}$ Departments of Neurology, ${ }^{6}$ Psychiatry, University of Pittsburgh, and

${ }^{7}$ Geriatric Research Education and Clinical Center, VA Pittsburgh Healthcare System, Pittsburgh, USA

Studies of acetylcholine degrading enzymes acetylcholinesterase (AChE) and butyrylcholinesterase (BChE) in Alzheimer's disease (AD) have suggested their potential role in the development of fibrillar amyloid- $\beta$ (A $\beta$ ) plaques (amyloid plaques). A recent genome-wide association study analysis identified a novel association between genetic variations in the $B C H E$ locus and amyloid burden. We studied BChE immunoreactivity in hippocampal tissue sections from AD and control cases, and examined its relationship with amyloid plaques, neurofibrillary tangles (NFT), dystrophic neurites (DN) and neuropil threads (NT). Compared to controls, AD cases had greater BChE immunoreactivity in hippocampal neurons and neuropils in CA2/3, but not in the CA1, CA4 and dentate gyrus. The majority of amyloid plaques ( $>\mathbf{8 0} \%$, using a pan-amyloid marker $\mathbf{X}-34$ ) contained discrete neuritic clusters which were duallabeled with antibodies against BChE and phosphorylated tau (clone AT8). There was no association between overall regional $\mathrm{BChE}$ immunoreaction intensity and amyloid plaque burden. In contrast to previous reports, BChE was localized in only a fraction $(\sim \mathbf{1 0} \%)$ of classic NFT (positive for X-34). A similar proportion of BChE-immunoreactive pyramidal cells were AT8 immunoreactive. Greater NFT and DN loads were associated with greater BChE immunoreaction intensity in CA2/3, but not in CA1, CA4 and dentate gyrus. Our results demonstrate that in AD hippocampus, BChE accumulates in neurons and plaqueassociated neuritic clusters, but only in a small proportion

Correspondence: Katsuyoshi Mizukami, MD, PhD, Graduate School of Comprehensive Human Sciences, University of Tsukuba, 1-1-1 Tennodai, Tsukuba, Ibaraki 305-8575, Japan. Email: kmizukam@taiiku. tsukuba.ac.jp

Received 27 February 2015; revised and accepted 27 July 2015. of NFT. The association between greater neurofibrillary pathology burden and markedly increased BChE immunoreactivity, observed selectively in $\mathrm{CA} 2 / 3$ region, could reflect a novel compensatory mechanism. Since $\mathrm{CA} 2 / 3$ is generally considered more resistant to $\mathrm{AD}$ pathology, $\mathrm{BChE}$ upregulation could impact the cholinergic modulation of glutamate neurotransmission to prevent/reduce neuronal excitotoxicity in AD hippocampus.

Key words: alzheimer's disease, amyloid, butyrylcholinesterase, hippocampus, tau.

\section{INTRODUCTION}

Impaired cholinergic neurotransmission contributes to cognitive dysfunction in Alzheimer's disease (AD), thus current symptomatic therapies for mild-to-moderate AD aim to block the acetylcholine (Ach) degrading enzymes, acetylcholinesterase (AChE) and butyrylcholinesterase (BChE). The distribution and localization of $\mathrm{AChE}$ in normal aging and AD are well described; ${ }^{1,2}$ however, less is known about $\mathrm{BChE}$, despite its role as an important modulator of Ach metabolism. ${ }^{3-6} \mathrm{BChE}$ inhibition is clinically significant as it provides benefit to patients resistant to AChE-specific inhibitor therapy. ${ }^{7}$ Furthermore, BChE inhibition may have additional, disease-modifying effects as it influences brain $\beta$-amyloid $(\mathrm{A} \beta)$ peptide concentration and ameliorates $A \beta$-induced cognitive impairment. ${ }^{8} \mathrm{Re}$ cently, an association was reported between genetic variation in the $\mathrm{BChE}$ gene locus and $\mathrm{A} \beta$-containing amyloid pathology burden measured using florbetapir positron emission tomography (PET) imaging. ${ }^{9}$ These observations warrant continued and more detailed analyses of $\mathrm{BChE}$ in AD. The distribution and cellular localization of BChE in the hippocampus have been described using histochemical methods in tissue sections, ${ }^{5,10}$ but results from this procedure

(C) 2015 Japanese Society of Neuropathology

\begin{tabular}{|l|l|l|l|l|l|l|l|l|l|l|l|l|}
\hline S & \multicolumn{4}{|c|}{ Journal Code } & \multicolumn{5}{|c|}{ Article ID } & Dispatch: 10.08.15 & CE: \\
\cline { 2 - 4 } & $\mathrm{N}$ & $\mathrm{E}$ & $\mathrm{U}$ & $\mathrm{P}$ & 1 & 2 & 2 & 4 & 1 & No. of Pages: 11 & ME:
\end{tabular}


vary across laboratories. ${ }^{11}$ Furthermore, several studies, using biochemical and histochemical methods, reported increased $\mathrm{BChE}$ activity in $\mathrm{AD},{ }^{12,13}$ as well as an association between $\mathrm{BChE}$ histochemical reaction and major neuropathological lesions of $\mathrm{AD}$, amyloid-structured aggregates of fibrillar $A \beta$ in plaques (hereafter referred to as amyloid plaques) and phosphorylated, fibrillar tau amyloid in neurofibrillary tangles (NFT) ${ }^{14-16}$ To extend these investigations, the current post mortem immunohistochemical study used a BChE-specific antibody to analyze $\mathrm{BChE}$ protein localization, distribution and immunoreaction intensity in the hippocampus from normal and AD cases, and to investigate its relation to amyloid structures of $A \beta$ plaques and neurofibrillary pathology, targets of PET radioligands currently in use or under development as imaging biomarkers for $\mathrm{AD} .{ }^{17}$

\section{METHODS}

\section{Cases}

We examined 11 cases with clinical and neuropathological diagnoses of $\mathrm{AD}$ and 10 age-matched controls with no clinical history of dementia, from Choju Medical Institute, Fukushimura Hospital and Ishizaki Hospital in Japan

T1 (Table 1). Cases were free of neuropathological lesions other than $\mathrm{AD}$, except one AD case (\#11) with coexisting Lewy bodies in brain stem and amygdala bilaterally with no involvement of the neocortex of hippocampus, and one AD case (\#15) with isolated, small infarctions bilaterally in the thalamus. Clinical diagnosis of $\mathrm{AD}$ was based on
DSM-IV $^{18}$ and NINCDS/ADRDA ${ }^{19}$ criteria. Neuropathological diagnosis was determined by a neuropathologist. All AD cases were assigned a neuropathological diagnosis of "definite" $\mathrm{AD}$, while controls were "not AD" according to CERAD criteria. ${ }^{20}$ NFT pathology was staged according to Braak and Braak ${ }^{21}$ (Table 1). Review of medical records revealed that one subject (case \#14) had received donepezil that was discontinued 1 year before death, but none of the subjects in the study had received rivastigmine or galantamine.

\section{Tissue processing and histology procedures}

At autopsy, brains were fixed in $10 \%$ buffered formalin for 3 weeks. Coronal blocks of hippocampus were dissected at the level of the lateral geniculate nucleus, embedded in paraffin, sectioned at $5 \mu \mathrm{m}$, and processed for immunohistochemistry using previously published protocols ${ }^{22,23}$ and a rabbit polyclonal antibody against BChE (Aviva Systems Biology, catalogue \#ARP44208, lot \#QC14544, dilution: 1:10), generated against a synthetic immunogen corresponding to N-terminal amino acids (SSLHVYDGKFLARVERVIVVSMNYRVGALGFLALPGNPEAPGNMGLFDQQ) of butyrylcholinesterase with $100 \%$ homology to human BChE (http://www. avivasysbio.com/bche-antibody-n-terminal-region-arp44208t100.html). Immunohistochemical signal was abolished in preadsorption experiments (not shown) using synthetic human BChE (Aviva, catalogue \#AAP44208). Sections from all subjects in the study were processed simultaneously. At least three sections from each case were immunolabeled with the $\mathrm{BChE}$ antibody, and additional three sections from each

Table 1 Case demographic details

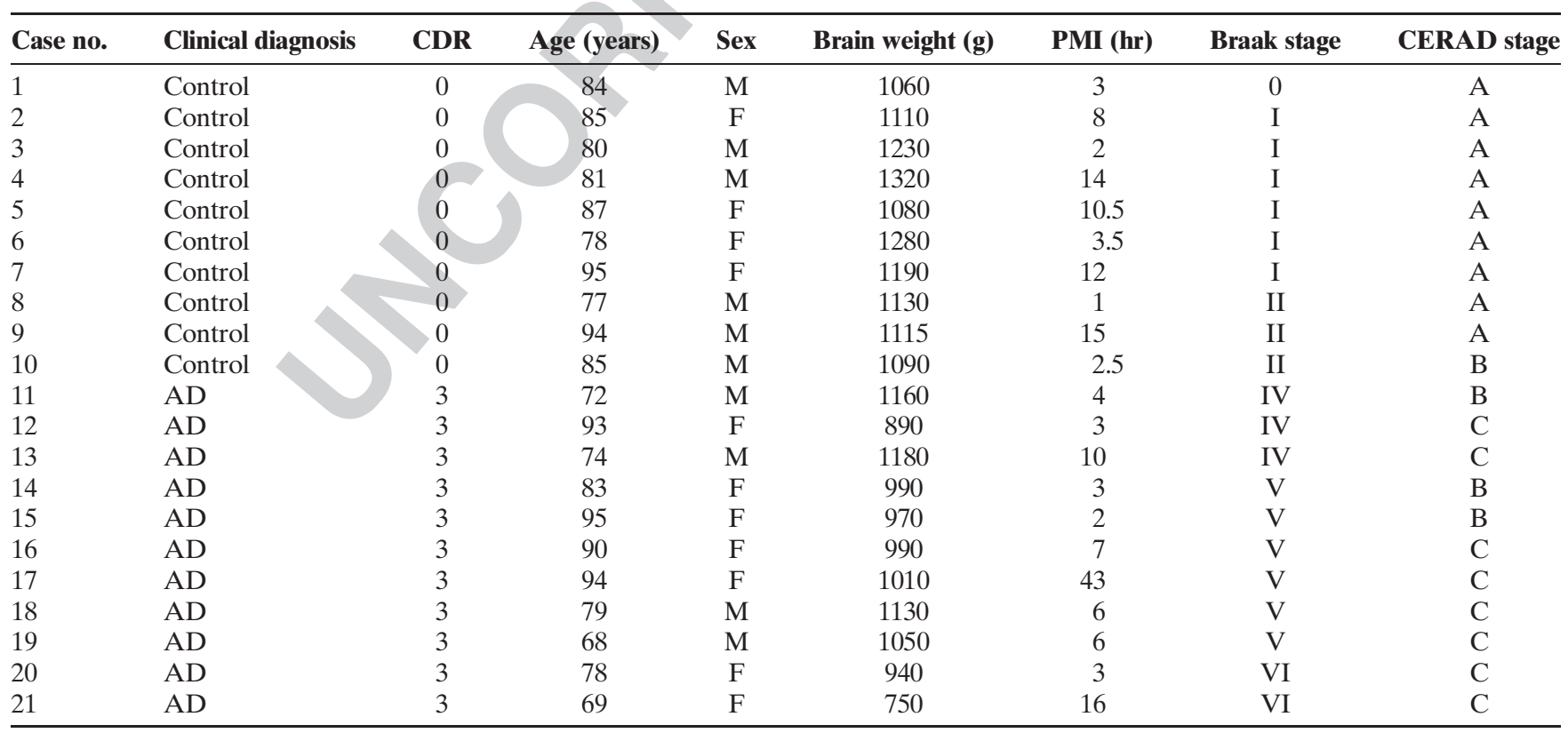

CDR, Clinical dementia rating; CERAD, Consortium to Establish a Registry for Alzheimer's Disease; PMI, post mortem interval. 
case were processed with cresyl violet to delineate the cytoarchitectural boundaries of the hippocampus as defined by Duvernoy. ${ }^{24}$

To assess the relation between $\mathrm{BChE}$ immunoreactivity and plaque and neurofibrillary amyloid pathology of $\mathrm{AD}$, BChE-immunoreacted sections were over-stained with the pan-amyloid dye X-34, a highly fluorescent derivative of Congo red that labels $\beta$-sheet structure of $\mathrm{A} \beta$ fibrils in plaques as well as tau fibrils in NFT, dystrophic neurites (DN), and neuropil threads (NT), as previously described. $^{25,26}$ To examine the association between BChE immunoreactivity and neurofibrillary pathology specifically, we performed dual immunofluorescence using an antibody against $\mathrm{BChE}$ (described above, dilution 1:20) and mouse

Q3 monoclonal antibody AT8 (The catalogue \#MN1020, lot \#00184107, dilution 1:3,000) that interacts with epitopes mapped to phosphorylated Ser202 and phosphorylated Thr205. Primary antibodies were visualized using fluorescent Alexa dyes conjugated to secondary antibodies Q4 generated against rabbit (Alexa 488 for BChE) and mouse (Alexa 594 for AT8). Immunofluorescently labeled sections were coverslipped using 4',6-diamino-2-phenylindole Q5 (DAPI) containing media (Vectashield Hard Set, Vector, H-1500).

\section{Quantitative measures}

\section{BChE immunoreaction intensity}

We assessed $\mathrm{BChE}$ immunoreaction intensity by quantifying immunoreaction product optical density (OD), using previously published protocols, ${ }^{23}$ in dentate gyrus (DG) granule cells and molecular layers, CA4 cells and neuropils, and in stratum oriens, pyramidale (pyramidal neurons and neuropil) and radiata of CA1 and CA2/3. Images of neuropils in AD cases were obtained using systematic random sampling and therefore included areas with varying presentations of BChE-immunoreactive plaques and surrounding, unaffected neuropils. For each case, three sections were imaged and in each subfield three images were taken using an Olympus BX53 microscope, equipped with an Olympus DP72 digital camera and Olympus cellSens Standard image capture Q6 software, using a UPlanSApo $10 \times$ objective (N.A. $=0.40$; Olympus, Tokyo, Japan). Illumination intensity was held constant throughout image acquisition. Images were assessed for grayscale intensity using public domain image analysis software (Rasband, W.S., ImageJ, United States National Institutes of Health, Bethesda, MD, USA). X-34 and immunofluorescence was visualized using an Olympus BX53 microscope equipped with an $\mathrm{X}$-Cite series $120 \mathrm{Q}$ fluorescence illuminator and DAPI, fluorescein isothiocyanate (FITC), tetramethylrhodamine (TRITC) and violet filters.

\section{Neurofibrillary and plaque amyloid pathology load}

We calculated percent area of tissue occupied by X-34labeled amyloid structure in plaques and each of three types of neurofibrillary pathology (NFT, DN and NT) in the same hippocampal regions assessed for $\mathrm{BChE}$ immunoreaction intensity. Images of X-34 stained sections were processed using the ImageJ threshold tool, and different types of X-34 positive amyloid pathology were identified as previously described. ${ }^{26}$ Briefly, amyloid plaques and NFT were distinguished based on their morphology (size and shape) and localization (extracellular or intracellular). DN were identified as X-34-positive dendritic swellings associated with plaques $(2-3 \mu \mathrm{m}$ diameter), while NT were X-34-positive smaller caliber $(\leq 1 \mu \mathrm{m}$ diameter) threads in the neuropil. Ghost tangles were identified as extracellular skeletons of advanced NFT.

\section{BChE and $X$-34 colocalization}

To assess the degree of colocalization/codistribution of BChE immunoreactivity with X-34-labeled amyloid structure of plaques and NFT, dual X-34/BChE labeled structures were quantified in each region of the hippocampus and reported as percent of X-34-labeled amyloid plaques or NFT containing BChE immunoreactivity.

\section{$B C h E$ and AT8 colocalization}

Colocalization/codistribution of $\mathrm{BChE}$ and AT8 immunofluorescence was assessed qualitatively in fields CA1, 2/3 and 4).

\section{Statistical analyses}

Individual values obtained for each case were combined to calculate the mean and standard error (SEM) for each group. In control and $\mathrm{AD}$ groups, we performed pairwise comparisons of $\mathrm{BChE}$ immunoreactivity or X-34-labeled pathology load in each examined subfield, using the Mann-Whitney $U$-test. Correlations of BChE immunoreaction intensity and X-34-labeled plaque and neurofibrillary pathology load were made using Spearman's $R$. All results are expressed as mean \pm SEM and $P<0.05$ was considered statistically significant.

\section{RESULTS}

Controls were cognitively normal (Clinical Dementia Rating $(\mathrm{CDR})=0)$ with Braak stages 0-II and CERAD score A (except for Case 10, CERAD score B, and no plaques in the hippocampus or $\mathrm{DG}$ ), while $\mathrm{AD}$ cases were cognitively impaired $(\mathrm{CDR}=3)$ with Braak stages IV-VI and CERAD score B or C (Table 1). Both case groups had similar mean age and post mortem intervals, but $\mathrm{AD}$ cases had lower mean brain weight than controls $(P<0.05)$. 


\section{BChE immunoreactivity in control and AD hippocampus}

In controls, $\mathrm{BChE}$ immunoreactivity was prominent in neurons in the granular and polymorphic layers of DG, hilus (CA4), and pyramidal layer in CA1 and CA2/3 fields, while

F2 F1 neuropil staining was low-to-moderate (Figs 1a,c and 2a,c). Neuronal BChE immunoreactivity was localized to the cytoplasm in cell bodies and proximal dendritic segment, while the nucleus was not labeled. We also observed $\mathrm{BChE} \mathrm{immu-}$ noreactivity in glial cells in the stratum oriens and in occasional BChE immunoreactive fibers traversing DG and CA4 (not shown). In AD hippocampus, the overall pattern of $\mathrm{BChE}$ immunoreactivity was similar to that observed in controls; however, neuronal and neuropil BChE immunoreactivity were more intense (Figs $1 \mathrm{~b}, \mathrm{~d}$ and $2 \mathrm{~b}, \mathrm{~d}$ ), particularly in region CA2/3 (Fig. 3, both $P<0.05$ ). In addition to neuronal and neuropil labeling, $\mathrm{AD}$ cases had discrete clusters of
F3
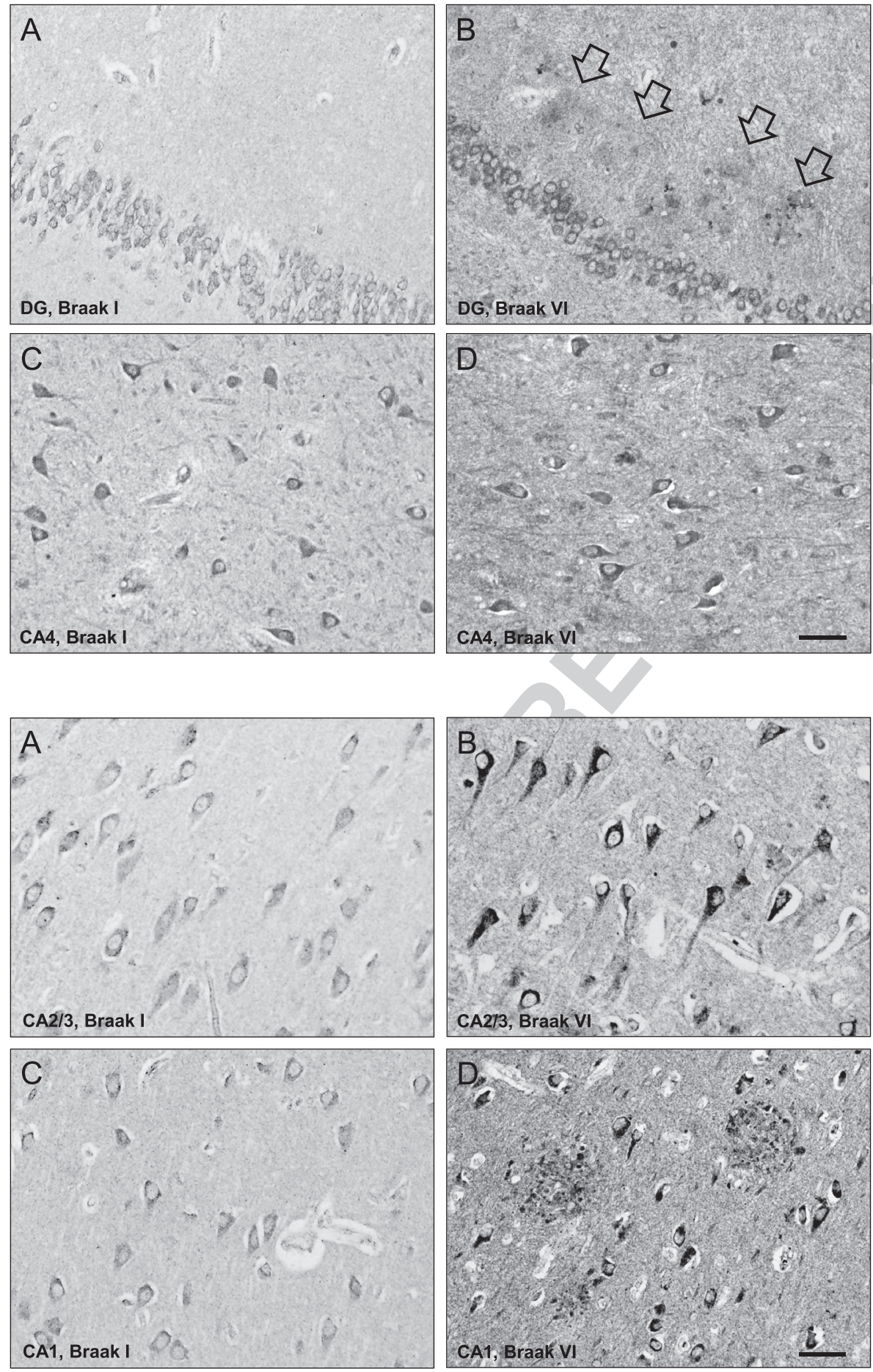

Fig. 1 Low-power photomicrographs of butyrylcholinesterase (BChE) immunoreactivity in the dentate gyrus (DG: A,B) and CA4 field (C,D) from representative Braak stage I control $(A, C)$ and a Braak stage VI AD case $(\mathrm{B}, \mathrm{D}$; arrows in $\mathrm{B}$ point at plaque-like clusters of $\mathrm{BChE}$ immunoreactive neuritic elements in DG molecular layer). Scale $\quad$ bar $=50 \mu \mathrm{m} \quad$ (A,B); $100 \mu \mathrm{m}(\mathrm{C}, \mathrm{D})$.
Fig. 2 High magnification of butyrylcholinesterase (BChE) immunoreactive cells and neuropil in pyramidal cell layer of CA2/3 (A,B) and CA1 (C,D) fields from representative Braak stage I control (A, C) and a Braak stage VI AD (B,D) case. Scale bar $=30 \mu \mathrm{m}(\mathrm{A}, \mathrm{B}) ; 50 \mu \mathrm{m}(\mathrm{C}, \mathrm{D})$. 
Fig. 3 Bar graph illustrating optical density measurements of butyrylcholinesterase (BChE) immunoreactivity in the hippocampus of Braak stage I-II control (empty bars) and Braak stage V-VI AD (solid bars) cases. DG, dentate gyrus; SG, stratum granulosum; SM, stratum moleculare; SO, stratum oriens; SP, stratum pyramidale; $\mathrm{SR}$, stratum radiatum. $* P<0.05 ; * * P<0.01$; *** $P<0.001$ (Mann-Whitney $U$-test).
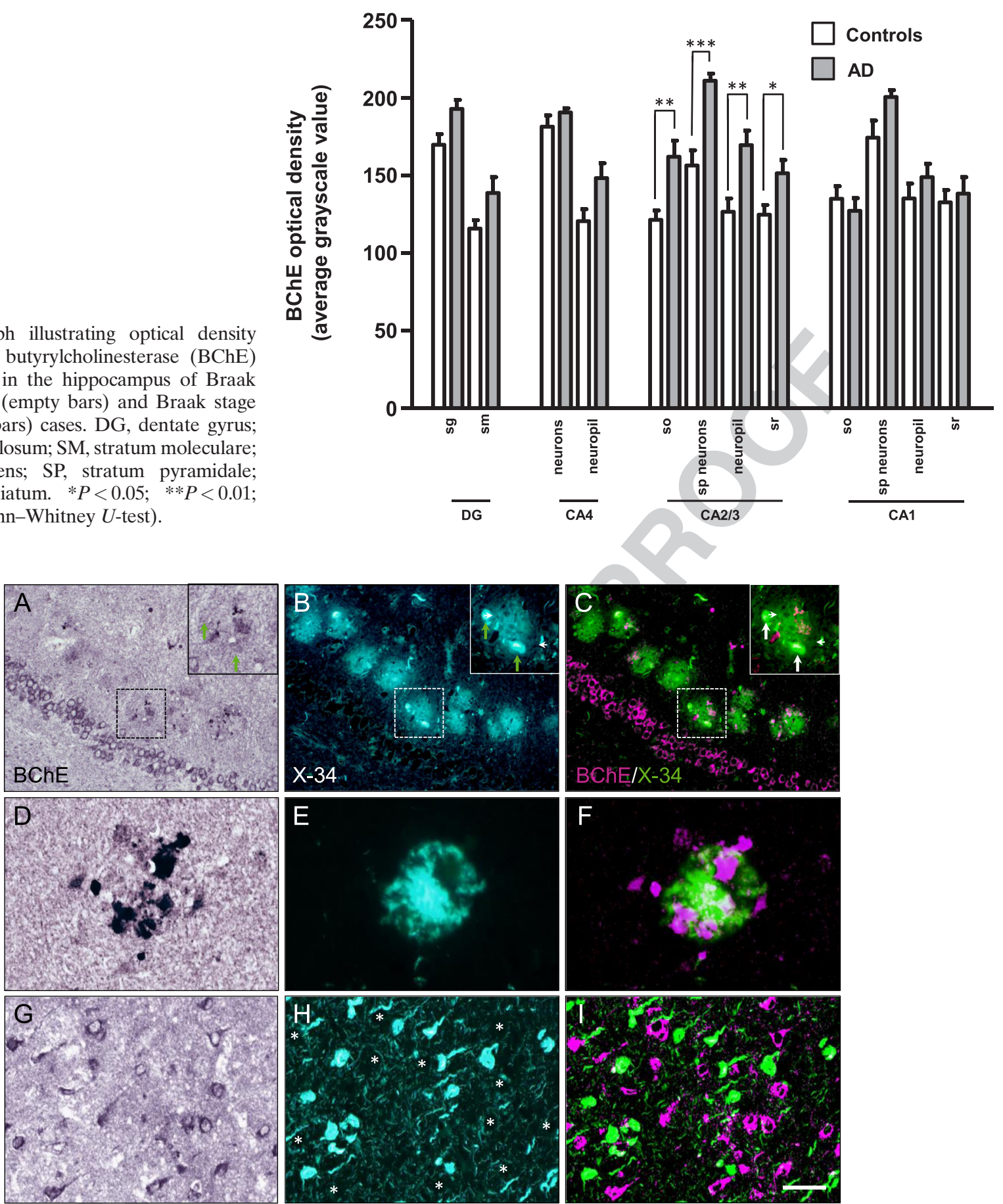

Fig. 4 Dual-labeling of butyrylcholinesterase $(B C h E)$ immunoreactivity $(A, D, G)$ and pan-amyloid $X-34$ fluorescence $(B, E, H)$ in the hippocampus of a Braak stage V AD case. Clusters of BChE immunoreactive neuritic swellings (A, D) are localized to X-34-labeled plaques $(B, E)$ in the dentate gyrus molecular layer $(A-C)$ and $C A 1$ region $(D-F)$. In panels A-C, the insets show at higher magnification the dashedoutlined plaque; BChE-immunoreactive neuritic swellings do not colocalize with X-34-labeled dystrophic neurites (arrows). In panels D-F, BChE-immunoreactive clusters are co-distributed, but do not co-localize, with X-34-labeled amyloid in plaque. Panels G-I illustrate lack of colocalization of BChE with X-34-labeled neurofibrillary tangles in CA1 (asterisks in $\mathrm{H}$ mark positions of BChE immunoreactive cells in $\mathrm{G})$. BChE and X-34 were pseudocolored and merged in (C, F and I), with BChE colored magenta and X-34 colored green. Scale bar $=100 \mu \mathrm{m}$ (A-C); $20 \mu \mathrm{m}(\mathrm{D}-\mathrm{F}) ; 50 \mu \mathrm{m}(\mathrm{G}-\mathrm{I})$.

BChE immunoreactivity in the DG molecular layer (Fig. 1b)

F4 and in CA fields (Fig. 4a-f). Quantitative analysis of dual label $\mathrm{X}-34 / \mathrm{BChE}$ revealed that these $\mathrm{BChE}$ immunoreactive clusters were localized to the majority $(>80 \%)$ of X-34 labeled amyloid plaques. Plaque-associated $\mathrm{BChE}$ positive clusters did not co-label with X-34 (Fig. 4a-c,d-f); however, 
they were immunoreactive for antibody clone AT8 (Fig. 5a-c). Only a minority ( $10 \%)$ of X-34-labeled NFT, primarily those in the $\mathrm{CA} 1$ region, contained $\mathrm{BChE}$ immunoreactivity (Fig. 4g-e). BChE and AT8 immunoreactivities did not colocalize in pyramidal cells (Fig. 5d-f,g-i). We also observed rare extracellular ghost tangles (large NFT lacking clearly pyramidal cell shape and without visible DAPI-stained nuclei), some of them resembling tangle-associated neuritic clusters (TANC). The intensity of BChE immunoreaction product in extracellular NFT was more prominent with a

F6 more advanced fibrillar structure revealed with X-34 (Fig. 6).

$\mathrm{X}-34$-positive amyloid plaque and neurofibrillary pathology loads ( $\%$ area occupied by plaques, NFT, NT or DN)

F7 were quantified separately in hippocampal fields (Fig. 7). Consistent with neuropathological diagnosis, measures of $\mathrm{X}$-34 fluorescent pathology loads were greater in AD than in controls (Fig. 7). There were several associations observed between X-34-labeled neurofibrillary pathology load measures (from Fig. 7) and BChE immunoreaction intensity measures (from Fig. 3). In the DG, greater X-34-labeled NT load correlated with greater BChE immunoreaction inten-

T2 sity in the granular cell layer (Table 2). In CA2/3, greater loads of X-34 labeled NFT and DN correlated significantly with greater intensity of BChE immunoreaction in all layers, and greater load of X-34-labeled NT correlated significantly with greater intensity of $\mathrm{BChE}$ immunoreaction in the pyramidal cell layer and in stratum oriens (Table 2). There were no statistically significant associations between X-34labeled neurofibrillary pathology load and BChE immunoreaction intensity in CA4 or CA1.

\section{DISCUSSION}

This study provides an antibody-based analysis of the distribution and localization of BChE in normal human hippocampus, and its alteration and relation to amyloid structure of plaques and neurofibrillary pathology in AD. While our method confirmed the presence of $\mathrm{BChE}$ in amyloid plaques, it also led to several novel findings, including: (i) $\mathrm{BChE}$ is not closely associated with classic NFT, in contrast to several prior reports; (ii) $\mathrm{BChE}$ immunohistochemistry is a viable alternative to enzyme histochemical methods used previously; and (iii) in AD hippocampus there is selective up-regulation of $\mathrm{BChE}$ in the $\mathrm{CA} 2 / 3$ region, generally considered more resistant to $\mathrm{AD}$ pathology.

The results of our immunohistochemical analyses of $\mathrm{BChE}$ extend previous studies which used histochemical detection of enzyme activity to examine BChE in aged humans and AD hippocampus. For example, using enzyme histochemistry, Darvesh and colleagues ${ }^{10}$ reported that $\mathrm{BChE}$ localized to neurons and their processes in the polymorphic layer of the DG, as well as stratum oriens and
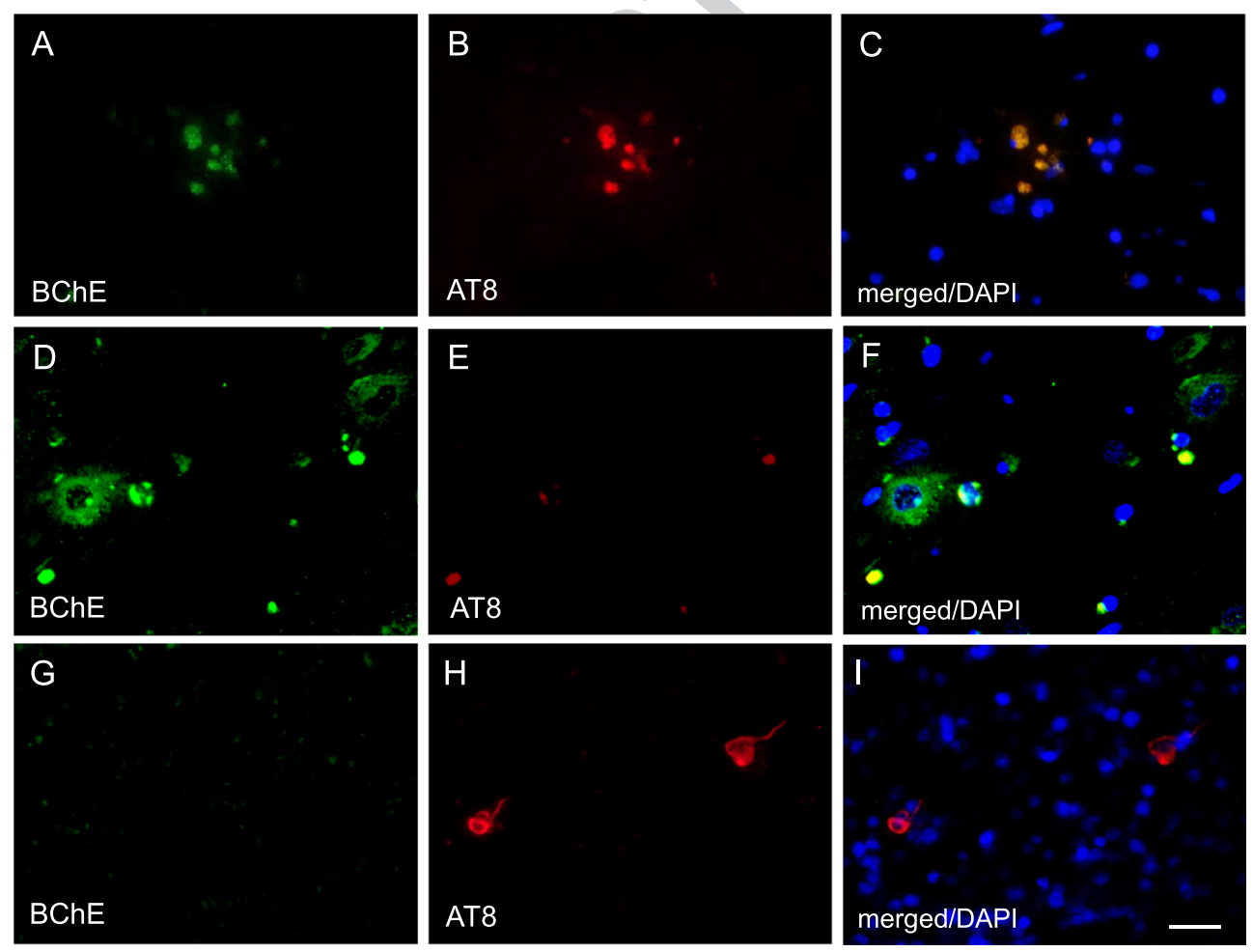

Fig.5 Dual immunofluorescence images showing overlapping butyrylcholinesterase (BChE) and AT8 immunoreactivity in plaque-associated neuritic clusters (A-C), and no overlap between BChE and AT8 immunoreactivity in CA1 pyramidal cells (D-F and G-I). Scale bar $=25 \mu \mathrm{m}(\mathrm{A}-\mathrm{F}), 35 \mu \mathrm{m}(\mathrm{G}-\mathrm{I})$. 

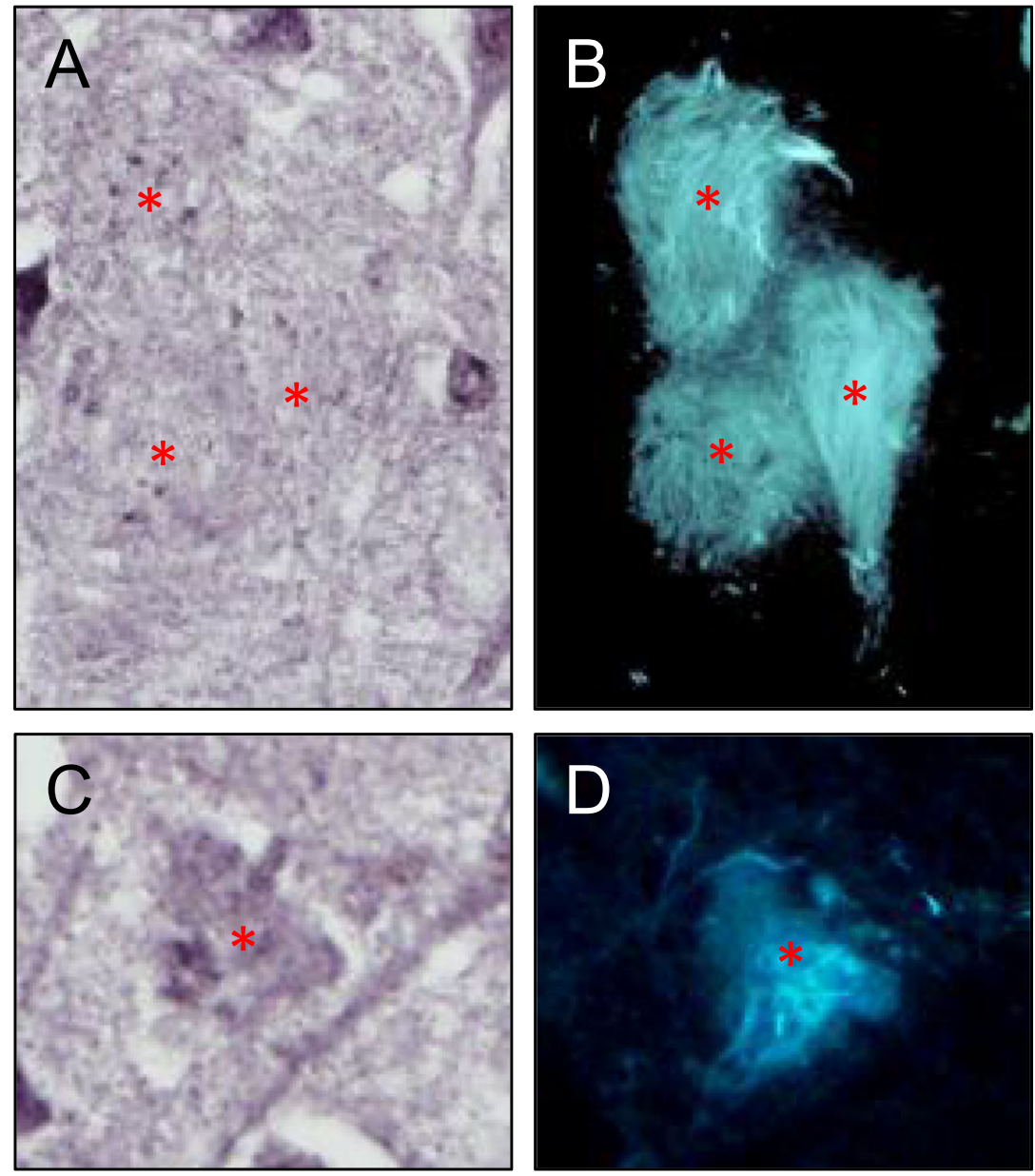

Fig. 6 Sections of an AD hippocampus (CA1 region) processed for butyrylcholinesterase $(\mathrm{BChE})$ immunohistochemistry (A,C,E) and over-stained with X-34 (B,D,F) demonstrate several types of extracellular ghost NFT (eNFT; B,D,F) with variable BChE immunoreactivity. BChE immunoreactivity is not discernable in eNFT with fine and cohesive fibrillary structure $(\mathrm{A}, \mathrm{B})$ while it is prominent in eNFT with a more coarse and dispersed fibrillary structure (C-F). Asterisks mark positions of the same eNFT in BChE and X-34 preparations. Scale bar $=15 \mu \mathrm{m}$.
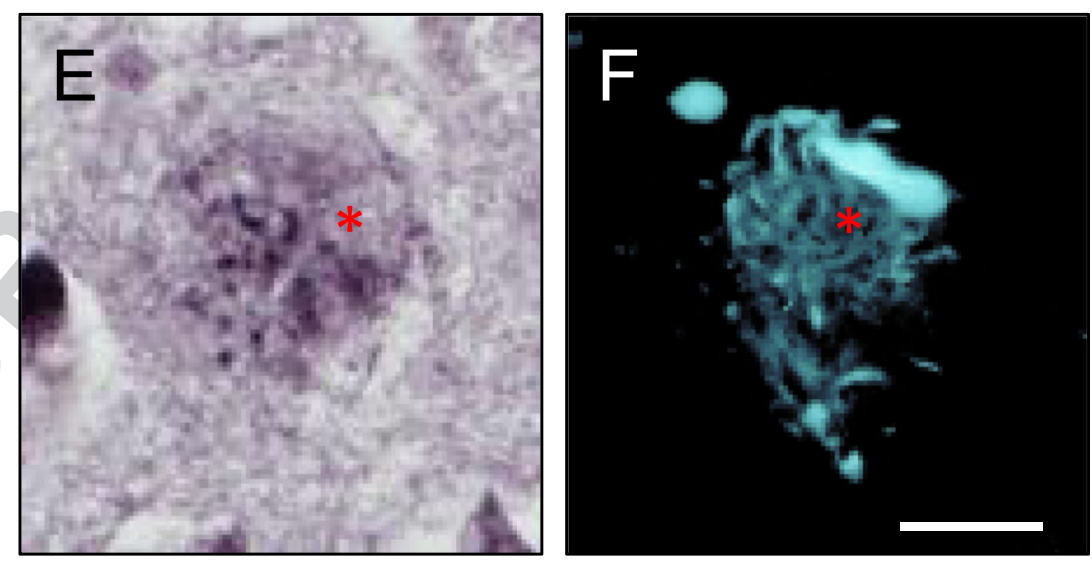

pyramidale of the CA fields, but not in the neuropils. While our study also demonstrates the preponderance of $\mathrm{BChE}$ in neuronal cells, we report that neuropils contain considerable $\mathrm{BChE}$ immunoreactivity in all hippocampal fields. The latter may be due to $\mathrm{BChE}$ containing glial cell processes, as suggested by Mesulam and colleagues. ${ }^{5}$ While we observed hippocampal BChE immunoreactive glial cell bodies in the stratum oriens of CA1-3 and in the white matter of the alveus, we were not able to resolve individual glial processes in the neuropil and we did not observe $\mathrm{BChE}$ (C) 2015 Japanese Society of Neuropathology immunoreactive glia in other hippocampal fields. In accord with our observation of $\mathrm{BChE}$ immunoreactive glial cells near myelinated fiber tracks in the hippocampus, Wright and colleagues ${ }^{27}$ reported that glia labeled with $\mathrm{BChE}$ enzyme histochemistry are abundant in deep cortical layers and white matter, in both control and AD brains. The functional significance of $\mathrm{BChE}$ in glia remains to be determined.

In $\mathrm{AD}$ cases, $\mathrm{X}-34$ positive amyloid plaques in the $\mathrm{DG}$ molecular layer and CA fields contained clusters of BChEimmunoreactive structures resembling ballooned axonal 


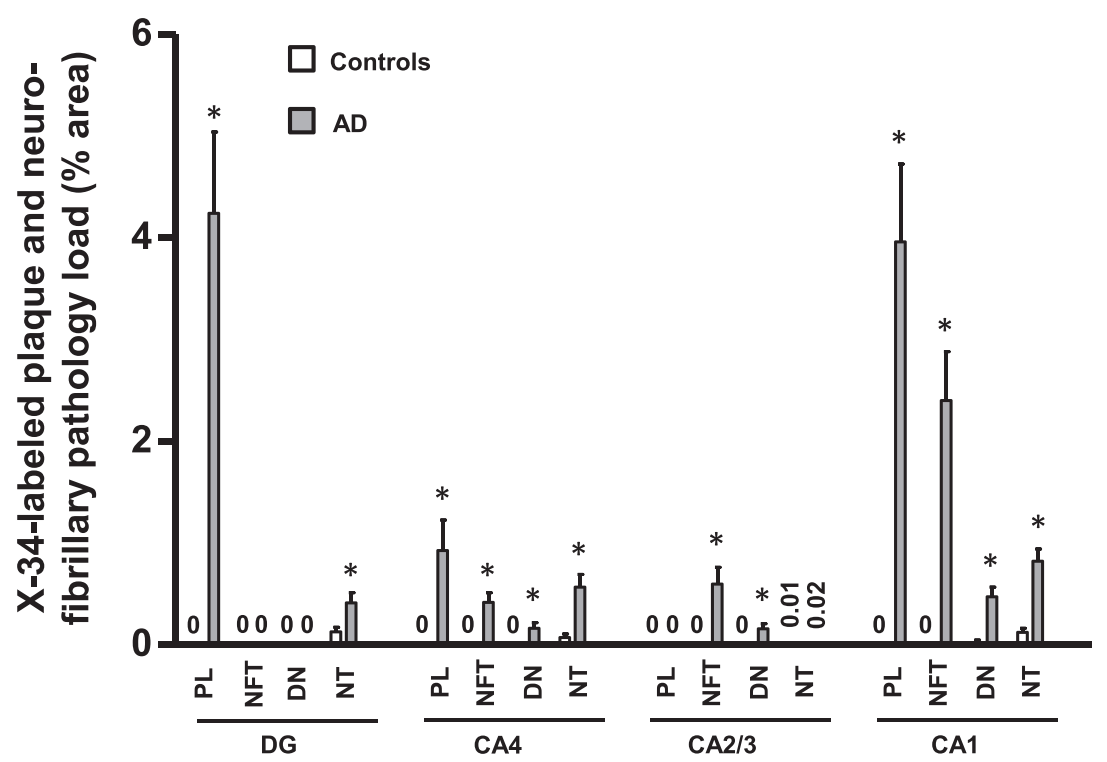

Fig. 7 Bar graph illustrating plaque and neurofibrillary pathology quantified by measuring percent area occupied by X-34-labeled structures in hippocampal fields of controls (empty bars) and AD (solid bars). DG, dentate gyrus; NFT, neurofibrillary tangles; DN, dystrophic neurites; NT, neuropil threads, PL, plaque load. $* P<0.05$.

Table 2 Correlation analyses of regional BChE immunoreaction intensity and X-34-labeled plaque, NFT, DN or NT pathology loads. Spearman's $R(P$-value, two tailed)

\begin{tabular}{|c|c|c|c|c|}
\hline \multirow{2}{*}{$\begin{array}{l}\text { Regional X-34 pathology load } \\
\text { DG or CA4 }\end{array}$} & \multicolumn{4}{|c|}{ BChE immunoreaction intensity (optical density in specific regional layers/structures) } \\
\hline & DG SG & DG SM & CA4 neurons & CA4 neuropil \\
\hline$\overline{\mathbf{P L}}$ & $0.46(0.13)$ & $0.29(0.36)$ & $0.48(0.13)$ & $0.55(0.06)$ \\
\hline DN & None present & None present & $0.59(0.054)$ & $0.58(0.046)$ \\
\hline NT & $0.76(0.0045)$ & $0.48(0.12)$ & $0.03(0.30)$ & $0.50(0.10)$ \\
\hline $\mathbf{C A} 2 / 3$ & CA2/3 SO & CA2/3 SP neurons & CA2/3 SP neuropil & CA2/3 SR \\
\hline NT & $0.62(0.033)$ & $0.69(0.014)$ & $0.53(0.079)$ & $0.31(0.33)$ \\
\hline CA1 & CA1 SO & CA1 SP neurons & CA1 SP neuropil & CA1 SR \\
\hline PL & $0.24(0.42)$ & $0.28(0.36)$ & $0.49(0.092)$ & $0.45(0.12)$ \\
\hline NFT & $0.28(.35)$ & $0.41(0.16)$ & $0.53(0.062)$ & $0.56(0.047)$ \\
\hline DN & $0.20(0.50)$ & $0.24(0.43)$ & $0.39(0.19)$ & $0.36(0.22)$ \\
\hline NT & $0.08(0.80)$ & $0.23(0.45)$ & $0.44(0.13)$ & $0.43(0.14)$ \\
\hline
\end{tabular}

BChE, butyrylcholinesterase; GDG, dentate gyrus (SG, stratum granulosum; SM, stratum moleculare); DN, dystrophic neurites; NFT, neurofibrillary tangles; NT, neuropil threads; PL, plaque load; SO, stratum oriens; SP, stratum pyramidale; SR, stratum radiatum. "Neuropil" refers to the neuropil surrounding neurons in CA fields $1,2 / 3$, and 4 .

terminals that were previously described as AChE-positive neurites in immature plaques. ${ }^{28}$ These BChE-containing clusters did not co-label with X-34, indicating lack of dense amyloid $\beta$-sheet structure; however, their co-labeling with antibody clone AT8 suggests they contain an early (pre-fibrillar) type of phosphorylated tau aggregate. In support of our current findings, a previous immunohistochemical study in $\mathrm{AD}$ and nondemented control cases demonstrated that at early stages of $\mathrm{A} \beta$ and thioflavin-S positive plaques, associated dystrophic neurites accumulating choline acetyltransferase and several neuropeptides lacked frank evidence of neurofibrillary pathology (i.e., immunoreactivity to Alz-50

Q7 or PHF antibodies ${ }^{29}$ ). Thus, BChE-immunoreactive clusters in plaques could belong to degenerating cholinergic fibers, similar to those described using AChE histochemistry in
$\mathrm{AD}$ cerebral cortex. ${ }^{29} \triangle$ The cholinergic origin of the BChEimmunoreactive clusters is additionally supported by reports of similar structures detected using AChE immunohistochemistry and histochemistry in aged monkey cerebral cortex ${ }^{30,31_{\curlywedge}}$ and $\mathrm{BChE}$ enzyme histochemistry in the $\mathrm{AD}$ brain. ${ }^{14,15,27,32,33_{\wedge}}$ Our observation that the majority $(>80 \%)$ of X-34 labeled amyloid plaques contained BChE is in agreement with previous reports.

We also observed a small proportion of diffuse, nonneuritic X-34 plaques without $\mathrm{BChE}$ immunoreactive clusters. This is in agreement with histochemical studies showing incomplete overlap of $\mathrm{AChE}$ and $\mathrm{BChE}$ with diffuse (thioflavin $\mathrm{S}$ negative) plaques. ${ }^{14}$ These and similar observations led Mesulam and colleagues to propose that plaque-associated $\mathrm{BChE}$ may have a role in the hypothetical (C) 2015 Japanese Society of Neuropathology 
conversion of diffuse plaques to compact plaques in $\mathrm{AD} \cdot{ }^{35_{\lambda}}$ This is a particularly compelling idea given the possibility that the enzymatic property of $\mathrm{BChE}$ in plaques differs from that in normal cells and axons ${ }^{27}$ and is pathologically significant, ${ }^{35_{\Lambda}}$ particularly since the enzyme influences APP processing $\frac{36}{\Lambda}$ and might contribute to $A \beta$ production and accumulation in AD. This is emphasized further by recently reported evidence from a genome-wide association study in the $\mathrm{ADNI}$ cohort, that variations at the $\mathrm{BChE}$ gene locus are associated with amyloid plaque load measured by florbetapir PET. 9

Despite the presence of $\mathrm{BChE}$ immunoreactivity in the majority of X-34-labeled amyloid plaques, we found no significant correlation between amyloid plaque load (\% area) and overall regional BChE OD intensity measures. This discrepancy could be explained by our observations that in the hippocampus: )i) $\mathrm{BChE}$ is predominantly localized to neurons and neuropils (regardless of plaque distribution); and (ii) BChE immunoreactivity in amyloid plaques is restricted to discrete clusters, whereas the entire plaque area occupied by $\beta$-sheet structure of amyloid (more dense in the compact core and less dense in the diffuse halo) is labeled with X-34. Additionally all AD cases in the present study had an advanced stage of dementia $(\mathrm{CDR}=3)$ with similarly heavy plaque burdens ( $\mathrm{CERAD}=\mathrm{B}$ or $\mathrm{C}$ ).

Similar to our observation of a poor co-localization of $\mathrm{BChE}$ and X-34-positive amyloid structure in dystrophic neurites, we found that $\mathrm{BChE}$ immunoreactivity colocalized with only a small proportion of X-34-positive intracellular and extracellular NFT (eNFT), some of them resembling TANC, ${ }^{37}$ in the AD hippocampus. These results are in agreement with several analyses of cholinesterases in eNFT which reported that cholinesterase labeling of eNFT varies at various stages of eNFT dissolution, ${ }^{15}$ and that cholinesterases are associated with $\mathrm{A} \beta$-immunoreactive, not tau, components of eNFT. ${ }^{16}$ The overall absence of AT8 signal in BChE immunoreactive neurons, suggests that tau protein in these cells has not yet fibrilized and, accordingly, is not detectable using amyloid-binding compounds such as X-34 (pre-tangles or early tangles). Future studies should employ a panel of antibodies against additional phosphorylated epitopes on tau, or different conformational states of the protein, to examine more closely the relationship between tau and BChE.

The finding that greater neurofibrillary pathology load correlated with higher $\mathrm{BChE}$ immunoreactivity in CA2/3, but not in CA1, warrants further investigation. This regional difference may reflect better resistance of $\mathrm{CA} 2 / 3$ neurons to fibrillary tau pathology in the $\mathrm{AD}$ brain. Up-regulated $\mathrm{BChE}$ could result in less ACh at mossy fiber terminals targeting CA2/3 pyramidal cells. Given the modulatory role of ACh in glutamate release, this response could fine-tune glutamate neurotransmission at the mossy fiber synapse, resulting in $\mathrm{CA} 2 / 3$ region-selective reduction in excitotoxicity. In contrast, plaque-associated $\mathrm{BChE}$ may have non-cholinergic functions and can be related to $A \beta$ containing amyloid pathology progression, as hypothesized by Geula and Mesulam.

In conclusion, we found that in $\mathrm{AD}$ hippocampus, $\mathrm{BChE}$ accumulating pyramidal cells and plaque-associated neuritic clusters do not contain dense amyloid structure typical of classic NFT and dystrophic neurites in neuritic plaques. However, in select areas the enzyme increase occurs in parallel with the progression of regional neurofibrillary pathology and therefore it may precede the development of amyloid aggregates or it could contribute to cells' resistance to such pathology. The functional consequences of these observations remain to be explored and could have important clinical implications for cholinergic (BChE targeted) therapies in AD.

\section{ACKNOWLEDGMENTS}

We are indebted to the subjects from Choju Medical Institute, Fukushimura Hospital and Ishizaki Hospital in this study. This work was supported by NIH grants NIA AG014449 and AG025204 (MDI), and by a Grant-in-Aid for Scientific Research from the Japanese Ministry of Education, Culture, Sports, Science and Technology (KM). Ms. Lan Shao, Ms. Natsuko Kato and Ms. Megumi Mitani provided expert technical assistance.

\section{REFERENCES}

1. Heckers S, Geula C, Mesulam MM. Acetylcholinesterase-rich pyramidal neurons in Alzheimer's disease. Neurobiol Aging 1992; 13: 455-460.

2. Mesulam MM, Geula C. Chemoarchitectonics of axonal and perikaryal acetylcholinesterase along information processing systems of the human cerebral cortex. Brain Res Bull 1994; 33: 137-153.

3. Geula C, Darvesh S. Butyrylcholinesterase, cholinergic neurotransmission and the pathology of Alzheimer's disease. Drugs Today (Barc) 2004; 40: 711-721.

4. Mesulam MM, Guillozet A, Shaw P, Levey A, Duysen EG, Lockridge O. Acetylcholinesterase knockouts establish central cholinergic pathways and can use butyrylcholinesterase to hydrolyze acetylcholine. $\mathrm{Neu}$ roscience 2002; 110: 627-639.

5. Mesulam MM, Guillozet A, Shaw P, Quinn B. Widely spread butyrylcholinesterase can hydrolyze acetylcholine in the normal and Alzheimer brain. Neurobiol Dis 2002; 9: 88-93.

6. Chatonnet A, Lockridge O. Comparison of butyrylcholinesterase and acetylcholinesterase. Biochem J 1989; 260: 625-634. 
7. Bartorelli L, Giraldi C, Saccardo M et al. Effects of switching from an AChE inhibitor to a dual AChE$\mathrm{BuChE}$ inhibitor in patients with Alzheimer's disease. Curr Med Res Opin 2005; 21: 1809-1818.

8. Greig NH, Utsuki T, Ingram DK et al. Selective butyrylcholinesterase inhibition elevates brain acetylcholine, augments learning and lowers Alzheimer beta-amyloid peptide in rodent. Proc Natl Acad Sci 2005; 102: 17213-17218.

9. Ramanan VK, Risacher SL, Nho K et al. APOE and $\mathrm{BCHE}$ as modulators of cerebral amyloid deposition: a florbetapir PET genome-wide association study. $\mathrm{Mol}$ Psychiatry 2014; 19: 351-357.

10. Darvesh S, Grantham DL, Hopkins DA. Distribution of butyrylcholinesterase in the human amygdala and hippocampal formation. J Comp Neurol 1998; 393: 374-390.

11. Morán MA, Gómez-Ramos P. Cholinesterase histochemistry in the human brain: effect of various fixation and storage conditions. J Neurosci Methods 1992; 43: 49-54.

12. Perry EK, Perry RH, Blessed G, Tomlinson BE. Changes in brain cholinesterases in senile dementia of Alzheimer type. Neuropathol Appl Neurobiol 1978; 4: 273-277.

13. Arendt T, Brückner MK, Lange M, Bigl V. Changes in acetylcholinesterase and butyrylcholinesterase in Alzheimer's disease resemble embryonic development-a study of molecular forms. Neurochem Int 1992; 21: 381-396.

14. Morán MA, Mufson EJ, Gómez-Ramos P. Colocalization of cholinesterases with beta-amyloid protein in aged and Alzheimer's brains. Acta Neuropathol 1993; 85: 362-369.

15. Morán MA, Mufson EJ, Gómez-Ramos P. Cholinesterases Colocalize with sites of neurofibrillary degeneration in aged and Alzheimer's brains. Acta Neuropathol 1994; 87: 284-292.

16. Cebrián JL, Morán MA, Gómez-Ramos P. Association of cholinesterases with amyloid in extracellular neurofibrillary tangles. Brain Res 1997; 19: 173-177.

17. Zwan MD, Okamura N, Fodero-Tavoletti MT et al. Voyage au bout de la nuit: $A \beta$ and tau imaging in dementias. $Q J$ Nucl Med Mol Imaging 2014; 58: 398-412.

18. American Psychiatric Association. Diagnostic and statistical manual of mental disorders IV. Washington, D.C.: American Psychiatric Association, 1994.

19. McKhann G, Drachman D, Folstein M, Katzman R, Price D, Stadlan EM. Clinical diagnosis of Alzheimer's disease: Report of Health and Human Services Task Force on Alzheimer's Disease. Neurology 1984; 34: 939-944.
20. Mirra SS, Heyman A, McKeel D et al. The consortium to establish a registry for Alzheimer's disease (CERAD). Part II. Standardization of the neuropathologic assessment of Alzheimer's disease. Neurology 1991; 41: 479-486.

21. Braak H, Braak E. Neuropathological stageing of Alzheimer-related changes. Acta Neuropathol 1991; 82: 239-259.

22. Iwakiri M, Mizukami K, Ikonomovic MD et al. An immunohistochemical study of GABA A receptor gamma subunits in Alzheimer's disease hippocampus: relationship to neurofibrillary tangle progression. Neuropathology 2009; 29: 263-269.

23. Mizukami K, Ishikawa M, Akatsu H, Abrahamson EE, Ikonomovic MD, Asada T. An immunohistochemical study of the serotonin $1 \mathrm{~A}$ receptor in the hippocampus of subjects with Alzheimer's disease. Neuropathology 2011; 31: 503-509.

24. Duvernoy HM. The Human Hippocampus. New York: Springer Verlag, 1988.

25. Styren SD, Hamilton RL, Styren GC, Klunk WE. X-34, a fluorescent derivative of Congo red: a novel histochemical stain for Alzheimer's disease pathology. $J$ Histochem Cytochem 2000; 48: 1223-1232.

26. Ikonomovic MD, Abrahamson EE, Isanski BA et al. X-34 labeling of abnormal protein aggregates during the progression of Alzheimer's disease. Methods Enzymol 2006; 412: 123-144.

27. Wright CI, Geula C, Mesulam MM. Neuroglial cholinesterases in the normal brain and in Alzheimer's disease: relationship to plaques, tangles, and patterns of selective vulnerability. Ann Neurol 1993; 34: 373-384.

28. Tago H, McGeer PL, McGeer EG. Acetylcholinesterase fibers and the development of senile plaques. Brain Res 1987; 406: 363-369.

29. Benzing WC, Ikonomovic MD, Brady DR, Mufson EJ, Armstrong DM. Evidence that transmittercontaining dystrophic neurites precede paired helical filament and Alz-50 formation within senile plaques in the amygdala of nondemented elderly and patients with Alzheimer's disease. J Comp Neurol 1993; 334 (2): 176-91.

30. McGeer EG, McGeer PL, Kamo H, Tago H, Harrop R. Cortical metabolism, acetylcholinesterase staining and pathological changes in Alzheimer's disease. Can J Neurol Sci 1986; 13: 511-516.

31. Kitt CA, Price DL, Struble RG et al. Evidence for cholinergic neurites in senile plaques. Science 1984; 226: 1443-1445.

32. Geula C, Mesulam MM. Special properties of cholinesterases in the cerebral cortex of Alzheimer's disease. Brain Res 1989; 498: 185-189.

(C) 2015 Japanese Society of Neuropathology 
33. Guillozet AL, Smiley JF, Mash DC, Mesulam MM. Butyrylcholinesterase in the life cycle of amyloid plaques. Ann Neurol 1997; 42: 909-918.

Q8 34. Eskander MF, Nagykery NG, Leung EY, Khelghati B, Geula C. Rivastigmine is potent inhibitor of acetyland butyrylcholinesterase in Alzheimer's plaques and tangles. Brain Res 2005; 1060: 144-152.

35. Mesulam MM, Geula C. Butyrylcholinesterase reactivity differentiates the amyloid plaques of aging from those of dementia. Ann Neurol 1994; 36: 722-727.

36. Small DH, Moir RD, Fuller SJ et al. A protease activity associated with acetylcholinesterase releases the membrane-bound form of the amyloid protein precursor of Alzheimer's disease. Biochemistry 1991; 30: $10795-10799$.

37. Munoz DG, Wang D. Tangle-associated neuritic clusters. A new lesion inAlzheimer's disease and aging suggests that aggregates of dystrophic neurites are not necessarily associated with beta/A4. Am J Pathol 1992; 140: $1167-1178$.

38. Geula C, Mesulam MM. Cholinesterases and the pathology of Alzheimer disease. Alzheimer Dis Assoc Disord 1995; 9: 23-28.

39. Struble RG, Cork LC, Whitehouse PJ, Price DL. Q9 Cholinergic innervation in neuritic plaques. Science 1982; 216: 413-415. 